\title{
Synergistic Inhibition of SARS-CoV-2 Replication using Disulfiram and Remdesivir
}

Ting Chen ${ }^{1 \#}$, Cheng-Yin $\mathrm{Fei}^{2}$, Yi-Ping Chen ${ }^{2}$, Karen Sargsyan ${ }^{1 \#}$, Chun-Ping Chang ${ }^{3}$, Hanna S. Yuan ${ }^{2 *}$, and Carmay $\operatorname{Lim}^{1,4 *}$

${ }^{1}$ Institute of Biomedical Sciences, Academia Sinica, Taipei 115, Taiwan

${ }^{2}$ Institute of Molecular Biology, Academia Sinica, Taipei 115, Taiwan

${ }^{3}$ Institute of Biotechnology and Pharmaceutical Research, National Health Research Institutes, Miaoli 350, Taiwan

${ }^{4}$ Department of Chemistry, National Tsing Hua University, Hsinchu 300, Taiwan

\section{KEYWORDS}

COVID-19, structural Zn-sites, nsp13, nsp14, replication and transcription complex, disulfiram, ebselen, remdesivir

\begin{abstract}
The SARS-CoV-2 replication and transcription complex (RTC) comprising nonstructural protein (nsp) 2-16 plays crucial roles in viral replication, reducing the efficacy of broad-spectrum nucleoside analog drugs such as remdesivir and in evading innate immune responses. Most studies target a specific viral component of the RTC such as the main protease or the RNA-dependent RNA polymerase. In contrast, our strategy is to target multiple conserved domains of the RTC to prevent SARS-CoV-2 genome replication and to create a high barrier to viral resistance or evasion of antiviral drugs. We show that clinically-safe Znejector drugs, disulfiram/ebselen, can target conserved $\mathrm{Zn}^{2+}$-sites in SARS-CoV-2 nsp13 and nsp14 and inhibit nsp13 ATPase and nsp14 exoribonuclease activities. As the SARS-CoV-2 nsp14 domain targeted by disulfiram/ebselen is involved in RNA fidelity control, our strategy allows coupling of the Zn-ejector drug with a broad-spectrum nucleoside analog that would otherwise be excised by the nsp14 proofreading domain. As proof-of-concept, we show that disulfiram, when combined with remdesivir, can synergistically inhibit SARS-CoV-2 replication in Vero E6 cells. We present a mechanism of action and the advantages of our multitargeting strategy, which can be applied to any type of coronavirus with conserved $\mathrm{Zn}^{2+}$-sites.
\end{abstract}




\section{INTRODUCTION}

The COVID-19 pandemic, caused by severe acute respiratory syndrome coronavirus 2 (SARSCoV-2), is affecting billions of people around the world. The recent approval of several vaccines against SARS-CoV-2 ${ }^{1}$ would help to reduce transmission. However, it is not known how long immunity stays and whether the vaccinated person, though protected from COVID19, may still carry the SARS-CoV-2 for some period and possibly infect others. To treat hospitalized COVID-19 patients, antivirals such as small-molecule drugs and neutralizing antibodies targeting SARS-CoV-2 are needed. Neutralizing antibodies derived from convalescent plasma obtained from a COVID-19 patient have shown only moderate success in clinical studies. ${ }^{2}$ De novo antiviral development is lengthy and costly. A shortcut approach is to repurpose widely available, inexpensive drugs that can be easily administered against SARSCoV-2. Most studies target a single SARS-CoV-2 protein ${ }^{3}$ such as the spike protein that mediates cell entry, ${ }^{4}$ the main protease $\left(\mathrm{M}^{\mathrm{pro}}\right)^{5,6}$ or the papain-like protease $\left(\mathrm{PL}^{\text {pro }}\right)^{7}$ that cleaves the viral polyprotein into its constituent proteins, or the RNA-dependent RNA polymerase (RdRp) that catalyzes viral RNA synthesis. ${ }^{8,9}$ However, the virus may evade anti-viral drugs that target a single viral protein since the spike receptor-binding domain is the most variable, ${ }^{10}$ whereas human proteases may substitute for viral proteases. One strategy is to create a high barrier to viral resistance/evasion of antiviral drugs by targeting invariant sites of the multiprotein SARS-CoV-2 replication and transcription complex (RTC) that enables viral replication and transcription (see below). Here, we show that two FDA-approved drugs (disulfiram and remdesivir) can synergistically inhibit SARS-CoV-2 replication by targeting conserved sites in multiple non-structural proteins (nsps) constituting the core of the RTC.

SARS-CoV-2 is an enveloped $\beta$-coronavirus containing a positive-sense single-stranded RNA (+ssRNA) genome that encodes 16 nonstructural, 4 structural and 9 accessory proteins. ${ }^{11}$ It infiltrates cells by binding to human cell surface receptors such as angiotensin-converting enzyme 2 (ACE2) via its spike glycoprotein, which upon cleavage by human proteases enables virus-cell membrane fusion (Figure 1A). ${ }^{3,12,13}$ Once inside, the viral RNA genome is released into the cytoplasm. Its 5 '-end is translated by the host protein synthesis machinery to replicase polyproteins (ppla and pplab) that are cleaved by virus-encoded proteases ( $\mathrm{M}^{\text {pro }}$ and $\mathrm{PL}^{\text {pro }}$ ) to 16 constituent nsps. ${ }^{14}$ Nsp2-16 form the viral RTC, ${ }^{15}$ which plays two crucial functions: (i) transcription of subsets of the viral genome to produce messenger RNAs that are translated into accessory and structural proteins, and (ii) replication of the full-length +ssRNA genome. The viral proteins are then assembled into a virion consisting of the spike (S), envelope (E), membrane $(\mathrm{M})$ and nucleocapsid $(\mathrm{N})$ proteins with the latter encapsidating the + ssRNA. ${ }^{15}$ From a single infected cell, thousands of virions leave. 
A

B

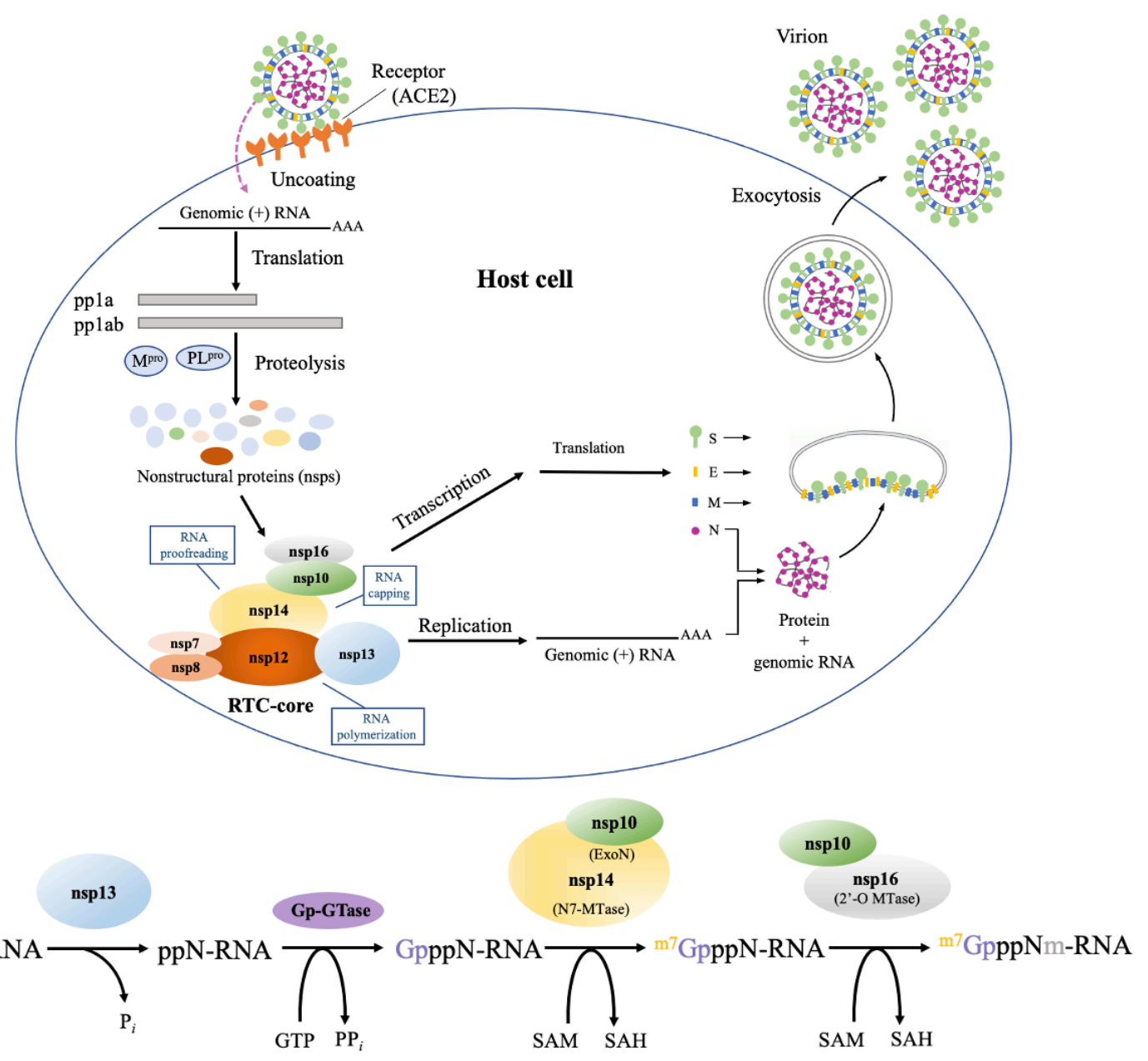

Figure 1. Importance of RTC in SARS-CoV-2 life cycle. (A) SARS-CoV-2 enters cells by binding its surface spike (S) protein to human ACE2 and possibly other cell receptors such as CD147. ${ }^{16}$ Once inside the cell, the $5^{\prime}$-end of its genomic RNA is translated by host ribosomes to yield viral nsps. Nsp7-8, nsp10, nsp12-14, and nsp16 form the core of the viral RTC, which drives the synthesis of full-length and subsets of the genomic ssRNA. (B) Capping of newly synthesized viral mRNA is initiated by nsp13 catalyzing the hydrolysis of the $5^{\prime}$ - $\gamma$-phosphate of the nascent pppN-RNA to ppN-RNA. A yet to be identified GTase transfers a GMP (Gp) to ppN-RNA yielding GpppN-RNA. Subsequently, the nsp14 N7-MTase methylates the guanosine N7 forming ${ }^{\mathbf{m} 7} \mathbf{G p p p N}$-RNA by demethylating co-enzyme S-adenosyl methionine (SAM) to S-adenosyl homocysteine (SAH). Finally, the nsp16 2'-O-MTase demethylates SAM to SAH and adds the methyl group to the ribose $2^{\prime}$-O, yielding ${ }^{\mathbf{m} 7} \mathbf{G p p p N m}$-RNA. ${ }^{17}$ nsp10 is needed to activate the enzymatic activities of nsp14 N7-MTase and nsp16 2'-O-MTase.

SARS-CoV-2 replication involves RNA synthesis, proofreading, and modification/capping. Viral RNA synthesis is catalyzed by the C-terminal RdRp domain of nsp12 with the help of nsp7 and nsp8 cofactors. ${ }^{8,18,19}$ RNA proofreading, the maintenance of the SARS-CoV-2 genome integrity, is carried out by the N-terminal $3^{\prime}-5^{\prime}$ exoribonuclease (ExoN) domain of nsp14, which recognizes erroneous nucleotides and catalyzes their 
excision. ${ }^{17}$ The nsp14 ExoN is stabilized by the nsp10 zinc-finger protein, which enhances its nucleolytic activity. ${ }^{20}$ Viral RNA capping, the addition of a cap structure to the newly synthesized viral mRNAs, ensures their efficient translation by host cell ribosomes and evasion of the host immune response. Without the cap structure, the viral RNA molecules are degraded and may be detected as "non-self" by the host, triggering innate immune responses. ${ }^{14}$ SARSCoV-2 mRNA cap synthesis involves nsp10 and four enzymes; viz., (i) nsp13 helicase-triphosphatase, (ii) an unknown guanylyltransferase (GTase), (iii) the nsp14 Cterminal (N7 guanine)-methyltransferase (N7-MTase) and (iv) the nsp16 2'-Omethyltransferase (2'-O-MTase). Nsp10 serves as an allosteric activator of the two methyltransferase enzymes and stabilizes conserved domains involved in fidelity control (nsp14) and mRNA capping (nsp13-16). ${ }^{14}:{ }^{17}$ Figure 1B summarizes the reactions involved in capping newly synthesized viral mRNA (pppN-RNA).

The viral proteins responsible for RNA synthesis, proofreading, and capping (Nsp7-8, nsp10, nsp12-14, and nsp16) form the RTC core. The following mechanism to produce stable viral RNA has been proposed: ${ }^{17,19}$ First, a RNA template is unwound by nsp13 helicase and subsequently translocated to the nsp12 active site where a nascent ssRNA is synthesized. ${ }^{19}$ The neighboring nsp14 excises any erroneous nucleotides from the nascent pppN-RNA, which is dephosphorylated by nsp13. An unknown GTase, nsp14, and nsp16 caps the viral ppN-RNA yielding ${ }^{\mathbf{m}} \mathbf{G p p p N m}$-RNA. nsp10, an allosteric activator of nsp14 and nsp 16, acts as a molecular connector between proofreading and capping activities. ${ }^{17}$ Because the RTC-core is indispensable for SARS-CoV-2 replication, using clinically safe drugs to target its constituent proteins would reduce viral load.

Interestingly, nsp3 PL pro domain, nsp10 zinc finger, nsp12, nsp13, and nsp14 possess several $\mathrm{Zn}^{2+}$-sites that are conserved among $\beta$-coronaviruses. ${ }^{21}$ The $\mathrm{Zn}^{2+}$-sites in these nsps serve an essential structural role ${ }^{18,21-24}$ as well as a vital catalytic role for the nsp13 helicase activity $^{24}$ and the nsp14 ExoN exoribonuclease activity. ${ }^{22}$ Among the multiple $\mathrm{Zn}^{2+}$-sites in SARS-CoV-2, the structural $\mathrm{Zn}^{2+}$-sites in nsp3 PL ${ }^{\text {pro }}$ and nsp10 have been found to be labile ${ }^{21}$ i.e., $\mathrm{Zn}^{2+}$ ions that contribute to structural stability are released upon reaction of the $\mathrm{Zn}^{2+}$-bound Cys thiolates with $\mathrm{Zn}^{2+}$-ejecting agents. ${ }^{25-28}$ However, it is not known whether the $\mathrm{Zn}^{2+}$-sites in nsp13 and nsp14, which constitute the RTC, are also labile and can be targeted by clinically safe $\mathrm{Zn}^{2+}$-ejector drugs such as disulfiram, an approved anti-alcoholic drug, and ebselen, in phase III clinical trials for hearing loss. To address this possibility, we have overexpressed SARS-CoV-2 nsp13 and nsp14 proteins to see if their $\mathrm{Zn}^{2+}$ ions could be ejected by $\mathrm{Zn}^{2+}$ ejecting agents, and if clinically safe disulfiram and ebselen can inhibit their enzymatic activities. Based on the finding herein that the $\mathrm{Zn}^{2+}$-ejector drugs could indeed inhibit the enzymatic activities of SARS-CoV-2 nsp13 and nsp14, we further assessed if disulfiram/ebselen combined with remdesivir, which stops RNA synthesis, could synergistically inhibit SARS-CoV-2 replication. 


\section{RESULTS}

\section{$\mathrm{Zn}^{2+}$ ions are released from SARS-CoV-2 nsp13 and nsp14 by $\mathrm{Zn}^{2+}$}

ejecting agents. The His-tagged full-length SARS-CoV-2 nsp13 and nsp14 were expressed in $E$. coli, and the recombinant proteins were purified by chromatographic methods to a high homogeneity. After incubating SARS-CoV-2 nsp13 and nsp14 with the $\mathrm{Zn}^{2+}$-specific fluorophore, FluoZin-3 $(1 \mu \mathrm{M})$, a $\mathrm{Zn}^{2+}$-ejecting compound $(5 \mu \mathrm{M})$ was added. Ten $\mathrm{Zn}^{2+}$ ejecting compounds were tested; viz., 2,2'-dithiobisbenzothiazole (also known as thiofide), cystamine dihydrochloride, 5,5'-dithiobis2-nitrobenzoic acid (DTNB), phenyl disulfide, 3nitrobenzamide, tetraethylthiuram disulfide (disulfiram), 2,2'-dithiodipyridine (aldrithiol-2), $\left( \pm\right.$ )alpha-lipoic acid, ebselen and cisplatin. Release of $\mathrm{Zn}^{2+}$ ions was monitored by the increase of the fluorescence signal from FluoZin-3 (Figure 2). Five of the $\mathrm{Zn}^{2+}$-ejecting compounds (DTNB, aldrithiol-2, disulfiram, thiofide, and ebselen) effectively ejected $\mathrm{Zn}^{2+}$ ions from the SARS-CoV-2 nsp13 and nsp14.

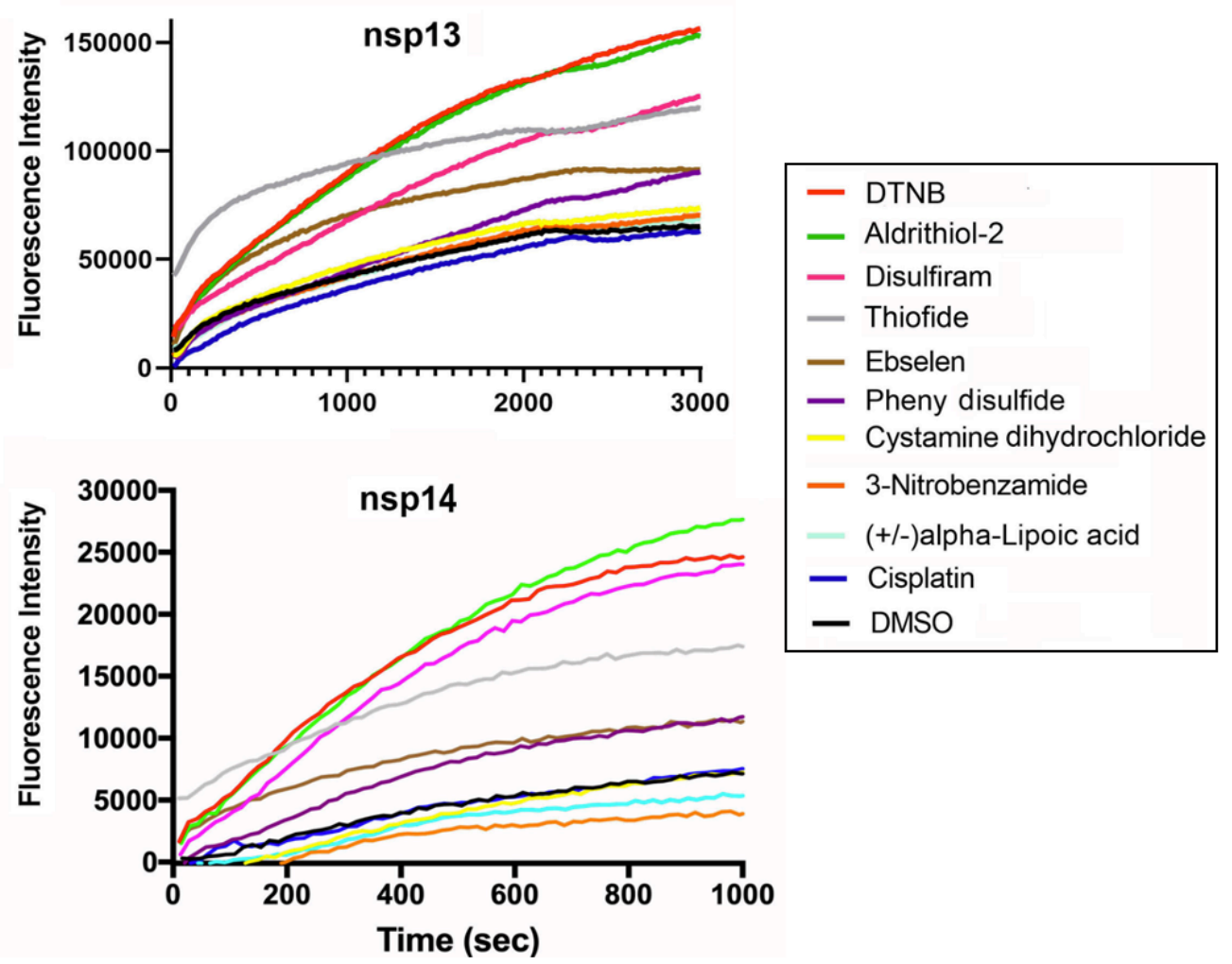

Figure 2. $\mathrm{Zn}^{2+}$ ions are released from SARS-CoV-2 nsp13 and nsp14 by $\mathrm{Zn}^{2+}$-ejecting compounds. The released $\mathrm{Zn}^{2+}$ ions were detected by the increase in the fluorescence signal from FluoZin-3 with emission and excitation wavelengths of $494 \mathrm{~nm}$ and $516 \mathrm{~nm}$, respectively.

Disulfiram and ebselen inhibit SARS-CoV-2 nsp13 ATPase activity. SARSCoV-2 nsp13 consists of an N-terminal $\mathrm{Zn}^{2+}$-binding domain that is connected to an inserted domain (1B) by a stalk region and two RecA ATPase domains. These domains work together to complete the helicase unwinding function. ${ }^{24,29}$ The $\mathrm{Zn}^{2+}$-binding domain is not directly 
involved in unwinding double-stranded DNA/RNA, but nevertheless, it is critical for nsp13 helicase activity. ${ }^{30}$ Can the release of $\mathrm{Zn}^{2+}$ ions from SARS-CoV-2 nsp13 affect its helicase activity? To address this, we exploited the fact that SARS-CoV-2 nsp13 is an NTP-dependent helicase and its unwinding activity/helicase activity depends on ATPase activity. ${ }^{24}$ Hence, we measured the ATPase activity of SARS-CoV-2 nsp13 by the molybdate method ${ }^{31}$ in the presence and absence of disulfiram and ebselen to examine if these $\mathrm{Zn}^{2+}$-ejecting compounds can inhibit nsp13 enzymatic activity. The relative ATPase activities of nsp13 were estimated based on the concentration of phosphate ions produced during ATP hydrolysis in the presence of single-stranded DNA and disulfiram $(0-12.5 \mu \mathrm{M})$ or ebselen $(0-10.0 \mu \mathrm{M})$ (Figure 3). Based on the measurements, disulfiram and ebselen inhibited the ATPase activity of nsp13 with a half maximal inhibitory activity ( $\mathrm{IC}_{50}$ ) of $410.7 \mathrm{nM}$ and $291.9 \mathrm{nM}$, respectively.
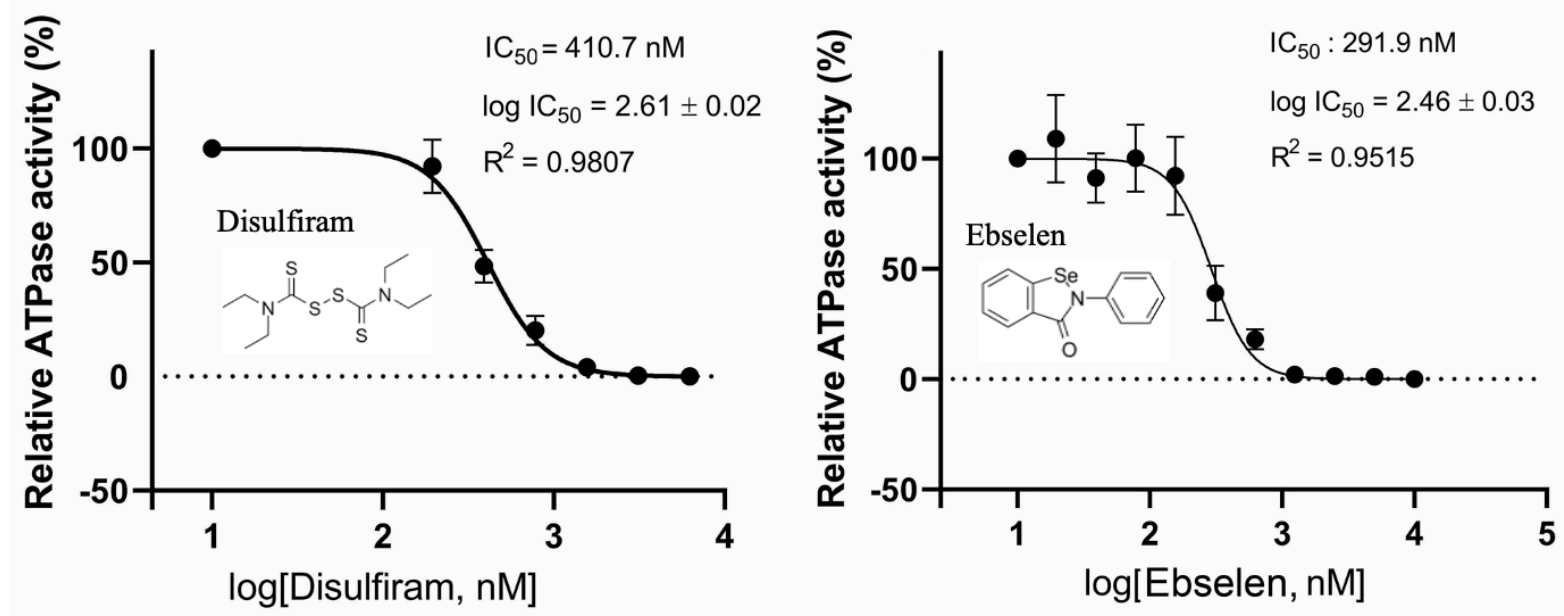

Figure 3. Inhibition of the ATPase activity of SARS-CoV-2 nsp13 by disufiram and ebselen. The ATPase activity of SARS-CoV-2 nsp13 $(0.25 \mu \mathrm{M})$ was estimated by measurement of the phosphate ion concentrations using molybdate method in the presence of a single-stranded DNA $(0.25 \mu \mathrm{M})$ and different concentrations of disulfiram $(0.2$ to $12.5 \mu \mathrm{M})$ or ebselen $(0.02$ to $10.0 \mu \mathrm{M})$. The half maximal inhibitory activity $\left(\mathrm{IC}_{50}\right)$ of disufiram and ebselen in inhibition of the ATPase activity of SARS-CoV-2 nsp13 is shown. Error bars shown in the two panels represent the standard errors from three replicates of the experiment.

\section{Disulfiram and ebselen inhibit SARS-CoV-2 nsp14 exoribonuclease}

activity. SARS-CoV nsp14 has two zinc-finger motifs (Zf1 and Zf2) in the ExoN domain responsible for proofreading and one zinc-finger motif (Zf3) in the N7-MTase domain involved in mRNA capping. ${ }^{22}$ Truncating the C-terminal region of Zf3 disrupted local hydrophobic interactions, causing a decrease in the N7-MTase activity. ${ }^{22}$ To see if release of $\mathrm{Zn}^{2+}$ cations from SARS-CoV-2 nsp14 could inhibit its exoribonuclease function, we measured the 3'-5' exoribonuclease activity of nsp14 in cleaving a 5'-end fluorophore-labeled ssRNA (5'-FAM$\left.\mathrm{C}_{7} \mathrm{U}_{30}-3^{\prime}\right)$ in the presence and absence of the $\mathrm{Zn}^{2+}$-ejecting compounds. The 37-nucleotide ssRNA was degraded by nsp14 into small fragments. However, upon addition of ebselen from 
0 to $100 \mu \mathrm{M}$ or disulfiram from 0 to $200 \mu \mathrm{M}$, the amount of cleaved RNA products was gradually reduced (Figure 4$)$. The relative (\%) nuclease activity with respect to different concentrations of a given $\mathrm{Zn}^{2+}$ ejector was calculated based on the band intensity of the cleaved products. This gave an estimated $\mathrm{IC}_{50}$ of $3.18 \mu \mathrm{M}$ for ebselen and $2.70 \mu \mathrm{M}$ for disulfiram in inhibiting SARS-CoV-2 nsp14 exoribonuclease activity.
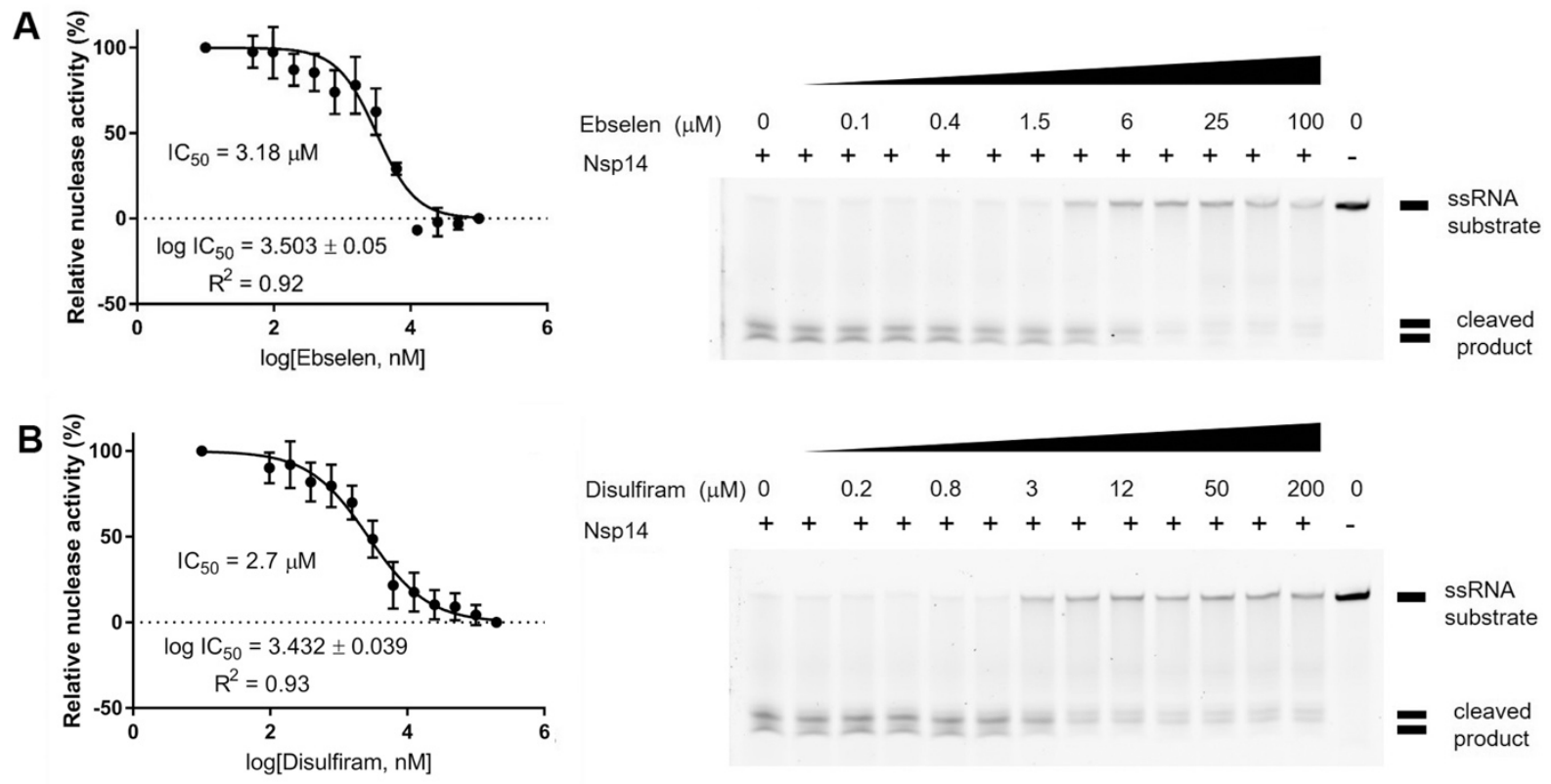

Figure 4. Inhibition of the exoribonuclease activity of SARS-CoV-2 nsp14 by ebselen and disulfiram. (A) SARS-CoV-2 nsp14 degraded the FAM-labeled 37-nucleotide ssRNA (5'-FAM- $\mathrm{C}_{7} \mathrm{U}_{30}-3^{\prime}$ ) into small fragments as revealed in the TBE gel (right panel). Increased concentrations of ebselen $(0-100 \mu \mathrm{M})$ gradually inhibited the nsp14 exoribonuclease activity with an estimated $\mathrm{IC}_{50}$ of $3.18 \mu \mathrm{M}$ (left panel). (B) Disulfiram inhibited the exoribonuclease activity of nsp14 with an estimated $\mathrm{IC}_{50}$ of $2.70 \mu \mathrm{M}$. In the two gels, the relative nuclease activity (\%) was estimated based on the band intensity of the cleaved products, normalized to the one in the first lane (no drug, 100\% activity) and that in the second last lane correspond $0 \%$ activity with $100 \mu \mathrm{M}$ ebselen or $200 \mu \mathrm{M}$ disulfirm. Error bars shown in the two left panels represent the standard errors from three replicates of the experiment.

Disulfiram and remdesivir exhibit synergistic antiviral activity. Remdesivir, an adenosine analog RdRp inhibitor, has gained emergency FDA-approval to treat acute COVID-19 patients. ${ }^{9,32}$ It outcompetes the natural ATP substrate for binding to nsp12 RdRp, and upon RNA chain elongation, its cyano $(\mathrm{CN})$ group has steric clashes with RdRp, inducing delayed chain termination. ${ }^{32-34}$ However, its efficacy is limited by the SARS-CoV-2 nsp14 ExoN domain, which can recognize and remove erroneous nucleotides (Figure 5A). ${ }^{35}$ Indeed, remdesivir has shown only moderate success in clinical studies: The ACTT-1 trial ${ }^{36}$ reported that remdesivir moderately reduced time to recovery, but not the WHO SOLIDARITY trial. ${ }^{37}$ Mortality results from four trials of remdesivir suggested some benefit in only low-risk patients. ${ }^{37}$ Since disulfiram/ebselen can inhibit SARS-CoV-2 nsp14 ExoN activity, this may 
allow remdesivir to be incorporated by the nsp 12 RdRp faster than its excision by the nsp 14 ExoN, thus leading to inhibition of viral replication.

A

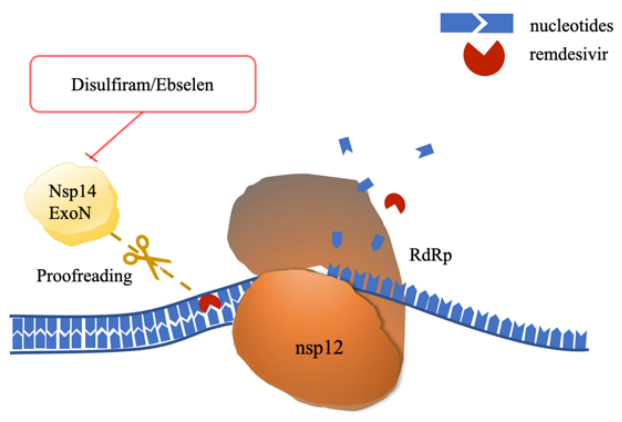

B
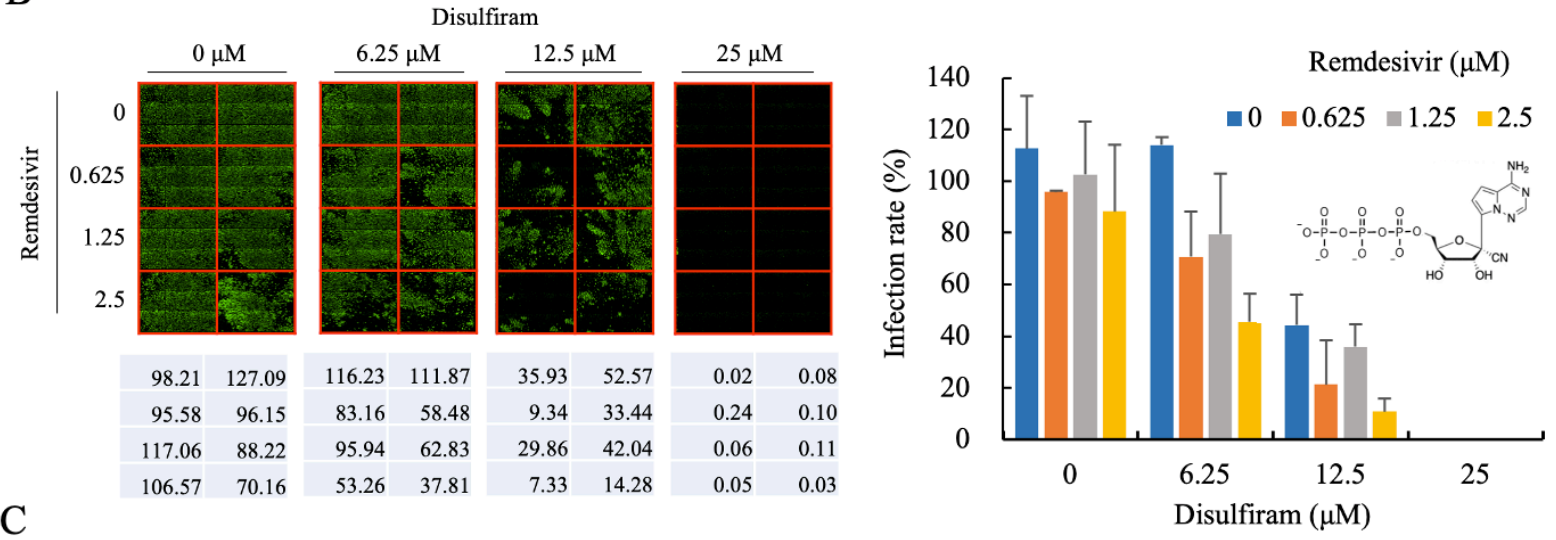

$\mathrm{C}$
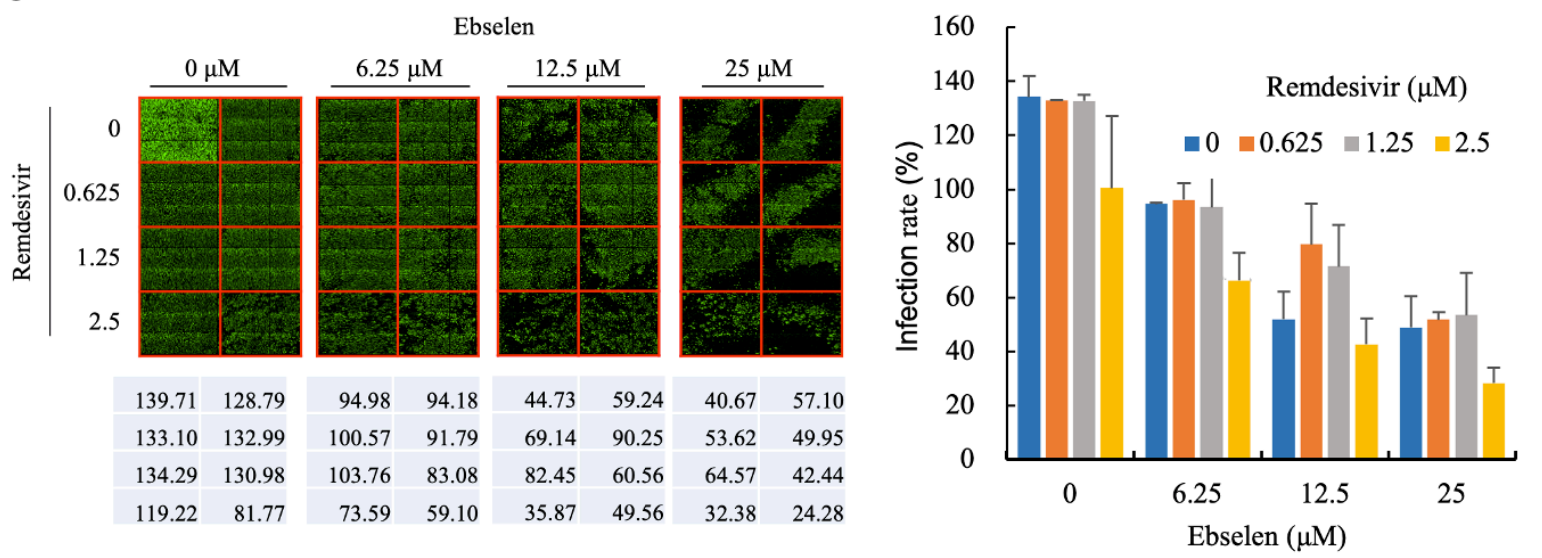

Figure 5. Synergistic antiviral potential of disulfiram/ebselen and remdesivir. (A) The active form of remdesivir inhibits nsp12 RdRp by competing with the natural ATP substrate. Disulfiram/ebselen can inhibit nsp14 ExoN activity, thus allowing remdesivir to inhibit viral RNA synthesis by nsp12 RdRp. A low-dosing window of synergistic antiviral effect in Vero E6 cells treated with remdesivir and (B) disulfiram or (C) ebselen was determined as described above, and shown as means and standard deviations $(n=2)$ on the right panel. The fluorescent signal (left panel) was quantified by high-content image analysis system (Molecular Devices). To detect SARS-CoV-2, the cells were stained with anti-SARS-CoV-2 N protein antibody and anti-human IgG-488 (shown in green). 
To see if remdesivir combined with disulfiram/ebselen could exhibit synergistic inhibition of SARS-CoV-2 replication, Vero E6 cells were treated with a given concentration of remdesivir and/or disulfiram/ebselen. Figure 5B and 5C shows the SARS-CoV-2 infection rate as the mean and corresponding standard deviation of two replicates. Disulfiram/ebselen combined with remdesivir exhibited enhanced antiviral effect compared to each drug alone. To quantify the synergistic antiviral effect, the synergy score between two drugs is calculated using the SynergyFinder. ${ }^{38}$ As there are different reference models for quantifying degrees of synergy and their synergy scores may sometimes disagree, we used several well-known models that are based on different assumptions (HSA, Bliss, Loewe, and ZIP) ${ }^{39}$. All four models gave consistent results, hence we report the ZIP score below. To make our prediction robust, we consider interaction between two drugs to be synergetic if the average ZIP score is $>10$, antagonistic if it is $<-10$, and additive if it is between -10 and 10 . The average ZIP score between disulfiram and remdesivir of 18.6 indicates synergistic interaction, whereas that between ebselen and remdesivir of 5.12 indicates only additive interaction. In addition to the average ZIP score, we also checked that the ZIP scores for every dose combination of disulfiram and remdesivir are also $>10$. The results verify that disulfiram and remdesivir exhibit synergistic SARS-CoV-2 inhibition for all doses studied.

\section{DISCUSSION}

The RTC-core, which plays critical roles in SARS-CoV-2 replication as well as evasion of nucleoside analog drugs and innate immune responses, is evidently an important drug target. To-date, drugs have been developed/repurposed to target a specific component of this complex such as the nsp12 RdRp. ${ }^{3,40}$ In sharp contrast, we employ clinically safe $\mathrm{Zn}^{2+}$-ejector drugs to target multiple conserved sites in the RTC-core. We show that disulfiram/ebselen can target conserved $\mathrm{Zn}^{2+}$-sites in nsp13 and nsp14 and when combined with remdesivir can synergistically/additively inhibit SARS-CoV-2 replication in Vero E6 cells. We propose the following mechanism for the observed effects: By ejecting $\mathrm{Zn}^{2+}$ from the $\mathrm{nsp} 13 \mathrm{Zn}^{2+}$-binding domain, which is crucial for helicase and triphosphatase activities and interactions with nsp12, ${ }^{24,29}$ disulfiram/ebselen not only inhibits nsp13 enzymatic activities, but may also disrupt nsp12---nsp13 interactions and affect nsp12 RdRp-catalyzed synthesis of viral RNA. It may also affect virus-host interactions since many human proteins involved in organization of the centrosome and Golgi, transcriptional regulation, protein kinase A and immune signaling, and vesicle trafficking have been identified to interact with nsp13 using affinity purification of 26 SARS-CoV-2 proteins followed by mass spectrometry. ${ }^{41}$ Notably, nsp13 targets innate immune signaling proteins along the interferon and nuclear factor- $\kappa \mathrm{B}$ pathways. ${ }^{41,42}$ On the other hand, by ejecting "structural" $\mathrm{Zn}^{2+}$ ions from the nsp14 ExoN domain, disulfiram/ebselen inhibits SARS-CoV-2 nsp14 exoribonuclease activity. This would mitigate the efficacy of nsp14 to excise erroneous nucleotides and allow nucleotide/nucleoside analog drugs such as 
remdesivir to inhibit viral RNA synthesis. In addition to nsp13 and nsp14, disulfiram/ebselen can also eject "structural" $\mathrm{Zn}^{2+}$ from the conserved nsp10 zinc finger domain, ${ }^{21}$ which would destabilize the nsp10-nsp14-nsp16 complex that is essential for mRNA capping (see Figure 1). ${ }^{14,43,44}$ This would allow the host's antiviral sensors to detect viral uncapped RNA, which can be degraded and prevent the virus from evading host immune responses.

Altogether, this work and previous studies show that disulfiram/ebselen can target multiple conserved sites in the viral RTC that is crucial for SARS-CoV-2 RNA genome replication as well as translation of the viral proteins (Figure 1). It can destabilize nsp10 cofactor, ${ }^{21}$ a crucial mediator of protein-protein interactions, and inhibit the enzyme activities of nsp3 PL pro, ${ }^{21}$ nsp5 $\mathrm{M}^{\text {pro }},{ }^{6}$ nsp13 and nsp14, and probably nsp12 and nsp16 through their interactions with nsp13 and nsp10-nsp14, respectively. Note that disulfiram/ebselen can not only target the $\mathrm{Zn}^{2+}$-bound cysteines, but also catalytic cysteines, ${ }^{21}$ and thereby inhibit SARS$\mathrm{CoV}-2 \mathrm{M}^{\text {pro }}{ }^{6}$ which does not possess a $\mathrm{Zn}^{2+}$-site. By inhibiting $\mathrm{M}^{\text {pro }}$ and $\mathrm{PL}^{\text {pro }}$ viral proteolysis, disulfiram/ebselen can prevent efficient cleavage of the replicase polyproteins into component nsps. In case the virus produces resistance against these proteases, disulfiram/ebselen can also inhibit the RTC-core that is crucial for viral RNA synthesis, proofreading and capping, thus restoring remdesivir's ability to function as a delayed chain terminator. As SARS-CoV-2 nsp3, nsp5, nsp13, nsp14 enzymes target the interferon and/or NF- $\mathrm{B}$ pathway, ${ }^{41,42}$ their inhibition by disulfiram/ebselen may help to restore innate immune responses.

Unlike most studies that target a specific viral protein such as $\mathrm{M}^{\text {pro5,6 }}$ or the spike protein ${ }^{1,2}$ by developing potent (nanomolar) inhibitors or antibodies, our strategy simultaneously targets multiple conserved viral proteins responsible for viral polypeptide proteolysis as well as viral genome replication and viral protein translation using clinically safe $\mathrm{Zn}^{2+}$-ejector drugs. Hence, it has several advantages. Whereas a single viral drug target such as $\mathrm{M}^{\text {pro }}$ or the spike protein can undergo mutations to produce resistance against specific drugs, it would be less likely for the multiple conserved viral proteins that are jointly responsible for viral replication and transcription to simultaneously mutate and achieve drug resistance. Mutations of the conserved $\mathrm{Zn}^{2+}$-binding cysteines in nsp3, nsp10, and nsp12-14 that are targeted by disulfiram/ebselen would likely disrupt binding to $\mathrm{Zn}^{2+}$ cations that serve essential structural and/or catalytic roles in several SARS-CoV-2 proteins. Furthermore, disulfiram/ebselen target upstream of the SARS-CoV-2 life cycle (Figure 1) before thousands of replicated viruses leave the infected cell. Importantly, disulfiram is inexpensive, widely available, and easily administered and it may allow other broad-spectrum antivirals to synergistically inhibit a new virus, as shown herein. A third advantage is that our strategy is not specific to a particular SARS-CoV-2 variant or any type of coronavirus. This is because the proteins essential for RNA replication such as the RTC-core are the most conserved among coronaviruses. Indeed, analysis of the SARSCoV-2 gene sequences show least divergence in the gene encoding these conserved domains (https://nextstrain.org/ncov/global). Thus, our approach may prove useful not only for the 
current COVID-19 pandemic, but also in future coronavirus outbreaks where it may be deployed as a first line of defense in the absence of monoclonal antibodies and vaccines.

In summary, we show that clinically safe $\mathrm{Zn}^{2+}$-ejector drugs (disulfiram/ebselen) can target conserved functionally important $\mathrm{Zn}^{2+}$-sites in the multi-protein RTC and that FDAapproved disulfiram combined with remdesivir can synergistically inhibit SARS-CoV-2 replication in Vero E6 cells (Figure 6).

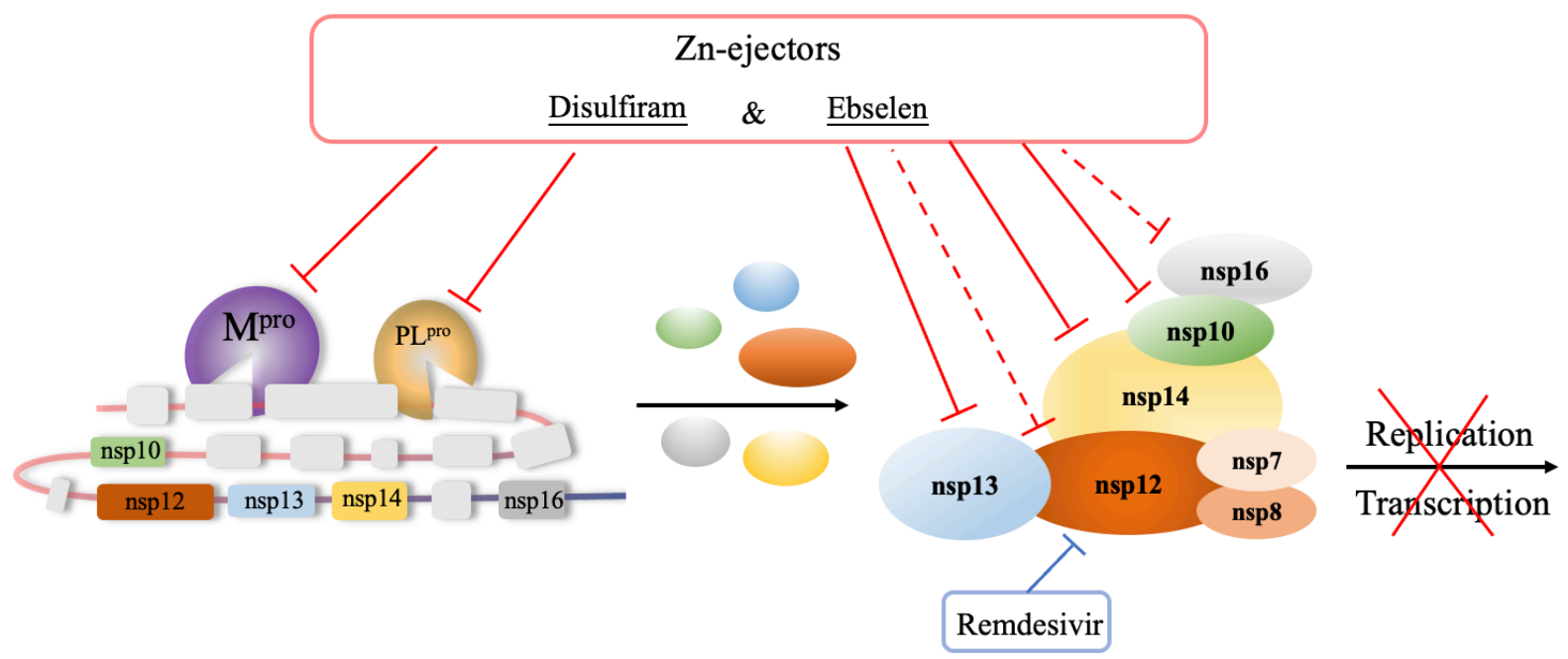

Figure 6. Disulfiram and ebselen can target multiple SARS-CoV-2 nsps to block the viral polyprotein cleavage and RNA replication/transcription. These $\mathrm{Zn}^{2+}$-ejector drugs target $\mathrm{PL}^{\text {pro }}$ of nsp3 and $\mathrm{M}^{\text {pro }}$ of nsp5 to inhibit viral polypeptide proteolysis. They also directly target nsp10, nsp13 and nsp14 as well as nsp12 and nsp16 indirectly to inhibit viral RNA genome replication and translation of the structural and accessory viral proteins. They can act in concert with remdesivir to synergistically inhibit SARS-CoV-2 replication. 


\section{EXPERIMENTAL SECTION}

Protein expression and purification. The cDNA of SARS-CoV-2 nsp 13 (residues 5302-5902 of pp 1a/pp1ab) and nsp14 (residues 5903-6429 pp1a/pp1ab) were synthesized from Protech, Inc. (Taiwan), and cloned respectively into pET28a and pRSFDuet-1 vectors for expression of the N-terminal His-tagged nsp13 and the C-terminal His-tagged nsp14. Both plasmids were transformed into E. coli BL21 (DE3) pLysS strain cultured in LB medium supplemented with $50 \mu \mathrm{g} / \mathrm{ml}$ kanamycin. Cells were grown to an optical density of 0.6 measured at a wavelength of $600 \mathrm{~nm}$ and induced by $0.2 \mathrm{mM} \mathrm{IPTG}$ at $18^{\circ} \mathrm{C}$ for $14-16 \mathrm{~h}$ to express the SARS-CoV-2 His-tagged nsp13 and nsp14.

The harvested cells with expressed nsp 13 were disrupted by a microfluidizer (Microfluidics M-110P) in lysis buffer containing $50 \mathrm{mM}$ Tris- $\mathrm{HCl}(\mathrm{pH} 8.0), 500 \mathrm{mM} \mathrm{NaCl}$, protease inhibitor, $5 \%$ glycerol and $10 \mathrm{mM} \beta$-mercaptoethanol. The supernatant was run through a HisTrap FF column ( $5 \mathrm{ml}$, GE HealthCare), and washed with a washing buffer containing $50 \mathrm{mM}$ Tris- $\mathrm{HCl}(\mathrm{pH} 8.0), 200 \mathrm{mM} \mathrm{NaCl}, 5 \%$ glycerol, and $30 \mathrm{mM}$ imidazole. The eluted nsp 13 was further purified by a HiTrap SP HP column ( $5 \mathrm{ml}$, GE HealthCare) to produce homogeneous nsp13 protein sample.

For the purification of nsp14, the harvested cells were resuspended in the lysis buffer (20 mM HEPES ( $\mathrm{pH}$ 7), $150 \mathrm{mM} \mathrm{NaCl}, 4 \mathrm{mM} \mathrm{MgCl}_{2}, 5 \%$ glycerol and $10 \mathrm{mM} \beta$ mercaptoethanol). After homogenization, the supernatant was loaded into a HisTrap FF column ( $5 \mathrm{ml}$, GE HealthCare), and washed with a washing buffer containing $20 \mathrm{mM}$ HEPES ( $\mathrm{pH} 7.0$ ), $150 \mathrm{mM} \mathrm{NaCl}, 4 \mathrm{mM} \mathrm{MgCl} 2,5 \%$ glycerol, $10 \mathrm{mM} \beta$-mercaptoethanol and $40 \mathrm{mM}$ imidazole. The protein sample was collected and further loaded into a gel filtration column (HiLoad 16/60 Superdex 200, GE HealthCare) and eluted by a buffer of $50 \mathrm{mM}$ HEPES (pH 7.4), $50 \mathrm{mM}$ $\mathrm{NaCl}, 5 \mathrm{mM} \mathrm{MgCl} 2$ and 5\% glycerol.

$\mathrm{Zn}^{2+}$ ejection assays. The $\mathrm{Zn}^{2+}$-ejecting agents were purchased from Sigma-Aldrich (USA), including 2,2'-dithiobis(benzothiazole), cystamine dihydrochloride, 5,5'-dithiobis(2nitrobenzoic acid) (DTNB), phenyl disulfide, 3-nitrobenzamide, tetraethylthiuram disulfide (disulfiram), 2,2'-dithiodipyridine, $\left( \pm\right.$ )alpha-lipoic acid, ebselen and cisplatin. The $\mathrm{Zn}^{2+}-$ specific fluorophore FluoZinTM-3 (Invitrogen/Life Technologies) was used to monitor the release of $\mathrm{Zn}^{2+}$ ions from SARS-CoV-2 nsp13 and nsp14. The $\mathrm{Zn}^{2+}$-ejecting agents were dissolved in DMSO to a stock solution of $100 \mu \mathrm{M}$. SARS-CoV-2 His-tagged nsp13 $(5 \mu \mathrm{M})$ and nsp14 $(5 \mu \mathrm{M})$ was respectively mixed with each $\mathrm{Zn}^{2+}$-ejecting agent $(5 \mu \mathrm{M})$ and FluoZin$3(1 \mu \mathrm{M})$ in a total reaction volume of $200 \mu \mathrm{l}$ at room temperature. Fluorescence emission was then measured by EnSpire Multilabel Plate Reader (Perkin Elmer, USA) at an excitation wavelength of $494 \mathrm{~nm}$ and emission wavelength of $516 \mathrm{~nm}$.

Inhibition of the nsp13 ATPase activity by $\mathrm{Zn}^{2+}$ ejector drugs. ATPase activity of SARS-CoV-2 nsp13 was analyzed by the molybdate method ${ }^{31}$ to measure the 
phosphate ion concentration in ATP hydrolysis reaction in the presence of the $\mathrm{Zn}^{2+}$ ejector drugs, disulfram and ebselen. Nsp13 $(2.5 \mu \mathrm{M}, 10 \mu \mathrm{l})$ was mixed with or without disulfiram (2 to $125 \mu \mathrm{M}, 10 \mu \mathrm{l})$ or ebselen $(0.2$ to $100 \mu \mathrm{M}, 10 \mu \mathrm{l})$ in $60 \mu \mathrm{l}$ of the reaction buffer containing $33 \mathrm{mM}$ Tris- $\mathrm{HCl}(\mathrm{pH} 8.0)$ and $5 \mathrm{mM} \mathrm{MgCl}_{2}$ at room temperature for 10 minutes. ATP $(50 \mathrm{mM}$, $10 \mu \mathrm{l})$ and ssDNA $(2.5 \mu \mathrm{M}, 10 \mu \mathrm{l})$ with a sequence of $5^{\prime}-(\mathrm{dT})_{24}-3^{\prime}$ were then added into the nsp13 solution and incubated for 10 mins. The ATPase reaction was stopped by adding $100 \mu 1$ of $10 \%$ SDS, and the blue complexes between inorganic phosphate and molybdate were developed by adding $100 \mu \mathrm{l}$ of $1.25 \%$ ammonium molydbate in $6.5 \%$ sulfuric solution and 100 $\mu \mathrm{l}$ of freshly prepared $10 \%(\mathrm{w} / \mathrm{v})$ ascorbic acid. The total of $400 \mu \mathrm{l}$ of reaction solution was incubated at $25^{\circ} \mathrm{C}$ for 10 mins and then read at $660 \mathrm{~nm}$ by Enspire plate reader (Perkin Elmer). Various concentrations of $\mathrm{Na}_{2} \mathrm{HPO}_{4}$ ranging from 0.05 to $0.5 \mathrm{mM}$ were also prepared as a standard curve. The relative ATPase activity of SARS-CoV-2 nsp13 in the presence of each $\mathrm{Zn}^{2+}$ ejector was compared to that without adding $\mathrm{Zn}^{2+}$ ejector, and the half maximal inhibitory activity $\left(\mathrm{IC}_{50}\right)$ of each inhibitor was calculated by GraphPad Prism.

Inhibition of the nsp14 exoribonuclease activity by $\mathrm{Zn}^{2+}$ ejector drugs. Nsp14 $(50 \mu \mathrm{M}, 100 \mu \mathrm{l})$ was mixed with $4 \mu \mathrm{l}$ of 5'-end FAM-labeled ssRNA ( $1 \mu \mathrm{M}, 5$ '-FAM$\left.\mathrm{C}_{7} \mathrm{U}_{30}-3^{\prime}\right)$ in $200 \mu \mathrm{l}$ of the reaction buffer (100 mM HEPES ( $\left.\mathrm{pH} 7.4\right), 100 \mathrm{mM} \mathrm{NaCl}, 10 \mathrm{mM}$

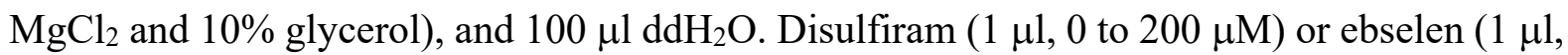
0 to $100 \mu \mathrm{M}$ ) in different concentrations was then added to the nsp14 and RNA mixture (19 $\mu \mathrm{l})$, and incubated for $30 \mathrm{mins}$ at $37^{\circ} \mathrm{C}$. The RNA loading dye $(20 \mu 1,89 \mathrm{mM}$ Tris-HCl, $\mathrm{pH} 8$, $89 \mathrm{mM}$ boric acid, $2 \mathrm{mM}$ EDTA, $0.01 \%$ bromophenol blue, $0.02 \%$ xylene cyanol FF and $7 \mathrm{M}$ Urea) was then added into the mixture and heated at $95^{\circ} \mathrm{C}$ for 5 mins to stop the reaction. The RNA in the mixtures were separated on TBE gel and visualized by Biomolecular Imager (Amersham Typhoon, GE HealthCare). The resolved RNA bands in the gel were analyzed by Image $\mathrm{J}$, and the $\mathrm{IC}_{50}$ of each inhibitor was calculated by GraphPad Prism.

Cell-based assays. Remdesivir was produced following the previous protocol. ${ }^{45}$ Vero E6 cells were pretreated with the disulfiram/ebselen and/or Remdesivir at various concentrations for $1 \mathrm{hr}$ at $37^{\circ} \mathrm{C}$ and then adsorbed with SARS-CoV-2 (TCDC\#4) at MOI 0.005 (100 PFU/well) for $1 \mathrm{hr}$ at $37^{\circ} \mathrm{C}$. After virus adsorption, the cells were replenished with fresh medium and compounds at the indicated concentrations for 1-day incubation. The cells were fixed with $10 \%$ formaldehyde, permeabilized with $0.5 \%$ Triton X-100, and stained with antiSARS-CoV-2 N protein antibody (provided by Dr. An-Suei Yang, Genomic Research Center, Academia Sinica, Taiwan) and anti-human IgG-488. The N protein expression was measured using a high-content image analysis system (Molecular Devices) and the average infection rate of no-drug treatment was set as $100 \%$. The synergy score is calculated using the SynergyFinder to quantify the synergistic antiviral effect between disulfiram/ebselen and Remdesivir. 


\section{AUTHOR INFORMATION}

\section{Corresponding Authors}

C. L. (carmay@gate.sinica.edu.tw)

H. Y. (hanna@sinica.edu.tw)

\section{ORCID}

Ting Chen: 0000-0001-8055-3104

Karen Sargsyan: 0000-0001-8931-6308

Hanna S. Yuan: 0000-0001-9671-6967

Carmay Lim: 0000-0001-9077-7769

\section{Notes}

The authors declare no competing financial interests.

\section{ACKNOWLEDGMENTS}

The cell-based experiments were performed by Dr. Jian-Jong Liang (jjliang1234@yahoo.com.tw) and Dr. Chun-Che Liao (jfliao@ibms.sinica.edu.tw) in Dr. YiLing Lin's laboratory at the Institute of Biomedical Sciences (IBMS), Academia Sinica, Taipei, Taiwan. We thank Taiwan CDC for providing SARS-CoV-2 (TCDC\#4) and funding support for the P3 facility in IBMS from Academia Sinica (AS-CFII-108-102) and the Ministry of Science and Technology, Taiwan (MOST 109-3114-Y-001-001). We thank Dr. Karine Mazmanian for help with the figures. We acknowledge the DNA Sequencing Core Facility (IBMS, AS-CFII-108-115) of the Scientific Instrument Center in Academia Sinica for DNA sequencing. This work was supported by the Ministry of Science \& Technology (MOST-107-2113-M-001-018 to C.L.) and Academia Sinica (AS-IA-107-L03 to C. L. and AS-IA-110-L02 to H.S.Y.) Taiwan.

\section{REFERENCES}

(1) Kim, P. S.; Read, S. W.; Fauci, A. S. Therapy for early COVID-19: A critical need. JAMA 2020, 324 (21), 2149-2150.

(2) Chen, P.; Nirula, A.; Heller, B.; Gottlieb, R. L.; Boscia, J.; Morris, J.; Huhn, G.; Cardona, J.; Mocherla, B.; Stosor, V. SARS-CoV-2 neutralizing antibody LYCoV555 in outpatients with Covid-19. N. Engl. J. Med. Published online Oct. 8, 2020.

(3) Zhou, Q. A.; Kato-Weinstein, J.; Li, Y.; Deng, Y.; Granet, R.; Garner, L.; Liu, C.; Polshakov, D.; Gessner, C.; Watkins, S. Potential therapeutic agents and associated bioassay data for COVID-19 and related human coronavirus infections. ACS Pharmacol. Transl. Sci. 2020, 3, 813-834. 
(4) Wrapp, D.; Wang, N.; Corbett, K. S.; Goldsmith, J. A.; Hsieh, C.-L.; Abiona, O.; Graham, B. S.; McLellan, J. S. Cryo-EM structure of the 2019-nCoV spike in the prefusion conformation. Science 2020, 367 (6483), 1260-1263.

(5) Zhang, L.; Lin, D.; Sun, X.; Curth, U.; Drosten, C.; Sauerhering, L.; Becker, S.; Rox, K.; Hilgenfeld, R. Crystal structure of SARS-CoV-2 main protease provides a basis for design of improved $\alpha$-ketoamide inhibitors. Science 2020, 368, 409-412.

(6) Jin, Z.; Du, X.; Xu, Y.; Deng, Y.; Liu, M.; Zhao, Y.; Zhang, B.; Li, X.; Zhang, L.; Peng, C.et al. Structure of $\mathrm{M}^{\text {pro }}$ from COVID-19 virus and discovery of its inhibitors. Nature 2020, 582, 289-293.

(7) Maiti, B. K. Can papain-like protease inhibitors halt SARS-CoV-2 replication? ACS Pharmacol. Transl. Sci. 2020, 3, 1017-1019.

(8) Gao, Y.; Yan, L.; Huang, Y.; Liu, F.; Zhao, Y.; Cao, L.; Wang, T.; Sun, Q.; Ming, Z.; Zhang, L.et al. Structure of the RNA-dependent RNA polymerase from COVID-19 virus. Science 2020, 368, 779-782.

(9) Yin, W.; Mao, C.; Luan, X.; Shen, D.-D.; Shen, Q.; Su, H.; Wang, X.; Zhou, F.; Zhao, W.; Gao, M. Structural basis for inhibition of the RNA-dependent RNA polymerase from SARS-CoV-2 by remdesivir. Science 2020, 368, 1499-1504.

(10) Matheson, N. J.; Lehner, P. J. How does SARS-CoV-2 cause COVID-19? Science 2020, 369 (6503), 510-511.

(11) Coronaviridae Study Group of the International Committee on Taxonomy of Viruses. The species Severe acute respiratory syndrome-related coronavirus: classifying 2019nCoV and naming it SARS-CoV-2. Nat. Microbiol. 2020, 5, 536-544.

(12) Letko, M.; Marzi, A.; Munster, V. Functional assessment of cell entry and receptor usage for SARS-CoV-2 and other lineage B betacoronaviruses. Nat. Microbiol. 2020, $5,562-569$.

(13) Hoffmann, M.; Kleine-Weber, H.; Schroeder, S.; Krüger, N.; Herrler, T.; Erichsen, S.; Schiergens, T. S.; Herrler, G.; Wu, N.-H.; Nitsche, A. SARS-CoV-2 cell entry depends on ACE2 and TMPRSS2 and is blocked by a clinically proven protease inhibitor. Cell 2020, 181 (2), 271-280.

(14) Snijder, E. J.; Decroly, E.; Ziebuhr, J. The nonstructural proteins directing coronavirus RNA synthesis and processing. Adv. Virus Res. 2016, 96, 59-126.

(15) V'kovski, P.; Kratzel, A.; Steiner, S.; H., S.; Thiel, V. Coronavirus biology and replication: implications for SARS-CoV-2. Nat. Rev. Microbiol. 2020, DOI:https://doi.org/10.1038/s41579-020-00468-6

(16) Wang, Y.; Grunewald, M.; Perlman, S. Coronaviruses: an updated overview of their replication and pathogenesis. Coronaviruses 2020, 1-29. 
(17) Romano, M.; Ruggiero, A.; Squeglia, F.; Maga, G.; Berisio, R. A structural view of SARS-CoV-2 RNA replication machinery: RNA synthesis, proofreading and final capping. Cells 2020, 9 (5), 1267.

(18) Kirchdoerfer, R. N.; Ward, A. B. Structure of the SARS-CoV nsp12 polymerase bound to nsp7 and nsp8 co-factors. Nat. Commun. 2019, 10 (1), 1-9.

(19) Yan, L.; Zhang, Y.; Ge, J. e. a. Architecture of a SARS-CoV-2 mini replication and transcription complex. Nat. Commun. 2020, 11, 5874.

(20) Bouvet, M.; Lugari, A.; Posthuma, C. C.; Zevenhoven, J. C.; Bernard, S.; Betzi, S.; Imbert, I.; Canard, B.; Guillemot, J. C.; Lécine, P.et al. Coronavirus Nsp10, a critical co-factor for activation of multiple replicative enzymes. J Biol. Chem. 2014, 289 (37), 25783-25796.

(21) Sargsyan, K.; Lin, C.-C.; Chen, T.; Grauffel, C.; Chen, Y.-P.; Yang, W.-Z.; Yuan, H. S.; Lim, C. Multi-targeting of functional cysteines in multiple conserved SARS-CoV2 domains by clinically safe Zn-ejectors. Chem. Sci. 2020, 11, 9904-9909.

(22) Ma, Y.; Wu, L.; Shaw, N.; Gao, Y.; Wang, J.; Sun, Y.; Lou, Z.; Yan, L.; Zhang, R.; Rao, Z. Structural basis and functional analysis of the SARS coronavirus nsp14nsp10 complex. Proc. Natl. Acad. Sci. USA 2015, 112 (30), 9436-9441.

(23) Hao, W.; Wojdyla, J. A.; Zhao, R.; Han, R.; Das, R.; Zlatev, I.; Manoharan, M.; Wang, M.; Cui, S. Crystal structure of Middle East respiratory syndrome coronavirus helicase. PLoS Pathog. 2017, 13, e1006474.

(24) Jia, Z.; Yan, L.; Ren, Z.; Wu, L.; Wang, J.; Guo, J.; Zheng, L.; Ming, Z.; Zhang, L.; Lou, Z.et al. Delicate structural coordination of the Severe Acute Respiratory Syndrome coronavirus Nsp13 upon ATP hydrolysis. Nucleic Acids Res. 2019, 47 (12), 6538-6550.

(25) Huang, M.; Maynard, A.; Turpin, J. A.; Graham, L.; Janini, G. M.; Covell, D. G.; Rice, W. G. Anti-HIV agents that selectively target retroviral nucleocapsid protein zinc fingers without affecting cellular zinc finger proteins. J. Med. Chem. 1998, 41 (9), 1371-1381.

(26) Briknarova, K.; Thomas, C. J.; York, J.; Nunberg, J. H. Structure of a zinc-binding domain in the Junin virus envelope glycoprotein. J. Biol. Chem. 2011, 286 (2), 1528-1536.

(27) Lee, Y. M.; Lin, Y. F.; Lim, C. Factors controlling the role of Zn and reactivity of Znbound cysteines in proteins: Application to drug target discovery. J. Chin. Chem. Soc. 2014, 61 (1), 142-150.

(28) Lee, Y. M.; Duh, Y.; Wang, S. T.; Lai, M. M. C.; Yuan, H. S.; Lim, C. Using an old drug to target a new drug site: Application of disulfiram to target the $\mathrm{Zn}$-site in $\mathrm{HCV}$ NS5A protein. J. Am. Chem. Soc. 2016, 138 (11), 3856-3862. 
(29) Chen, J.; Malone, B.; Llewellyn, E.; Grasso, M.; Shelton, P. M.; Olinares, P. D. B.; Maruthi, K.; Eng, E. T.; Vatandaslar, H.; Chait, B. T. Structural basis for helicasepolymerase coupling in the SARS-CoV-2 replication-transcription complex. Cell 2020, 182 (6), 1560-1573.

(30) Seybert, A.; Posthuma, C. C.; Van Dinten, L. C.; Snijder, E. J.; Gorbalenya, A. E.; Ziebuhr, J. A complex zinc finger controls the enzymatic activities of nidovirus helicases. J. Virol. 2005, 79 (2), 696-704.

(31) He, Z.; Honeycutt, C. W. A modified molybdenum blue method for orthophosphate determination suitable for investigating enzymatic hydrolysis of organic phosphates. Commun. Soil Sci. Plant Anal. 2005, 36 (9-10), 1373-1383.

(32) Eastman, R. T.; Roth, J. S.; Brimacombe, K. R.; Simeonov, A.; Shen, M.; Patnaik, S.; Hall, M. D. Remdesivir: A review of its discovery and development leading to emergency use authorization for treatment of COVID-19. ACS Central Science 2020, 6, 672-683.

(33) Badgujar, K. C.; Ram, A. H.; Zanznay, R.; Kadam, H.; Badgujar, V. C. Remdesivir for COVID-19: A review of pharmacology, mechanism of action, in-vitro activity and clinical use based on available case studies. J. Drug Delivery Therapeut. 2020, 10 (4s), 264-270.

(34) Gordon, C. J. e. a. Remdesivir is a direct-acting antiviral that inhibits RNA-dependent RNA polymerase from severe acute respiratory syndrome coronavirus 2 with high potency. J. Biol. Chem. 2020, 295, 6785-6797.

(35) Shannon, A.; Le, N. T. T.; Selisko, B.; Eydoux, C.; Alvarez, K.; Guillemot, J.-C.; Decroly, E.; Peersen, O.; Ferron, F.; Canard, B. Remdesivir and SARS-CoV-2: Structural requirements at both nsp12 RdRp and nsp14 Exonuclease active-sites. Antiviral Res. 2020, 104793.

(36) Beigel, J.; Tomashek, K.; Dodd, L. Remdesivir for the treatment of Covid-19 - final report. N. Engl. J. Med. 2020, 383, 1813-1826.

(37) WHO Solidarity Trial Consortiium. Repurposed antiviral drugs for COVID-19interim WHO SOLIDARITY trial results. N. Engl. J. Med. 2020, DOI:10.1056/NEJMoa2023184

(38) Ianevski, A.; Giri, A. K.; Aittokallio, T. SynergyFinder 2.0: visual analytics of multidrug combination synergies. Nucleic Acids Res. 2020, 48 (W1), W488-W493.

(39) Vlot, A. H.; Aniceto, N.; Menden, M. P.; Ulrich-Merzenich, G.; Bender, A. Applying synergy metrics to combination screening data: agreements, disagreements and pitfalls. Drug Discov. Today 2019, 24 (12), 2286-2298.

(40) Li, G.; Clercq, E. D. Therapeutic options for the 2019 novel coronavirus (2019nCoV). Nat. Rev. Drug Discov. 2020, 19, 149-150. 
(41) Gordon, D. E.; Jang, G. M.; Bouhaddou, M. e. a. A SARS-CoV-2 protein interaction map reveals targets for drug repurposing. Nature 2020, 583, 459-468.

(42) Lei, X.; Dong, X.; Ma, R.; Wang, W.; Xiao, X.; Tian, Z.; Wang, C.; Wang, Y.; Li, L.; Ren, L.et al. Activation and evasion of type I interferon responses by SARS-CoV-2. Nat. Commun. 2020, 11, 3810.

(43) Decroly, E.; Debarnot, C.; Ferron, F.; Bouvet, M.; Coutard, B.; Imbert, I.; Gluais, L.; Papageorgiou, N.; Sharff, A.; Bricogne, G.et al. Crystal structure and functional analysis of the SARS-coronavirus RNA cap 2'-O-methyltransferase nsp10/nsp16 complex. PLoS Pathog. 2011, 7 (5), e1002059.

(44) Pruijssers, A. J.; Denison, M. R. Nucleoside analogues for the treatment of coronavirus infections. Curr. Opin. Virol. 2019, 35, 57-62.

(45) Siegel, D.; Hui, H. C.; Doerffler, E.; Clarke, M. O.; Chun, K.; Zhang, L.; Neville, S.; Carra, E.; Lew, W.; Ross, B.et al. Discovery and synthesis of a phosphoramidate prodrug of a pyrrolo[2,1-f][triazin-4-amino] adenine C-nucleoside (GS-5734) for the treatment of Ebola and emerging viruses. J. Med. Chem. 2017, 60, 1648-1661. 